\title{
Intraoperative MRI-guided resection of focal cortical dysplasia in pediatric patients: technique and outcomes
}

\author{
Matthew F. Sacino, BS, ${ }^{1}$ Cheng-Ying Ho, MD, PhD, ${ }^{3}$ Jonathan Murnick, MD, PhD, ${ }^{2}$ \\ Tammy Tsuchida, MD, PhD, ${ }^{4}$ Suresh N. Magge, MD, ${ }^{1}$ Robert F. Keating, MD, ${ }^{1}$ \\ William D. Gaillard, MD, ${ }^{4}$ and Chima O. Oluigbo, MD $^{1}$ \\ Departments of ${ }^{1}$ Neurosurgery, ${ }^{2}$ Neuroradiology, ${ }^{3}$ Neuropathology, and ${ }^{4}$ Neurology, Children's National Medical Center, \\ Washington, DC
}

OBJECTIVE Previous meta-analysis has demonstrated that the most important factor in seizure freedom following surgery for focal cortical dysplasia (FCD) is completeness of resection. However, intraoperative detection of epileptogenic dysplastic cortical tissue remains a challenge, potentially leading to a partial resection and the need for reoperation. The objective of this study was to determine the role of intraoperative MRI (iMRI) in the intraoperative detection and localization of FCD as well as its impact on surgical decision making, completeness of resection, and seizure control outcomes.

METHODS The authors retrospectively reviewed the medical records of pediatric patients who underwent iMRI-assisted resection of FCD at the Children's National Health System between January 2014 and April 2015. Data reviewed included demographics, length of surgery, details of iMRI acquisition, postoperative seizure freedom, and complications. Postsurgical seizure outcome was assessed utilizing the Engel Epilepsy Surgery Outcome Scale.

RESULTS Twelve consecutive pediatric patients (8 females and 4 males) underwent iMRI-guided resection of FCD lesions. The mean age at the time of surgery was 8.8 years \pm 1.6 years (range 0.7 to 18.8 years), and the mean duration of follow up was 3.5 months \pm 1.0 month. The mean age at seizure onset was 2.8 years \pm 1.0 year (range birth to 9.0 years). Two patients had Type 1 FCD, 5 patients had Type 2A FCD, 2 patients had Type 2B FCD, and 3 patients had FCD of undetermined classification. iMRI findings impacted intraoperative surgical decision making in 5 (42\%) of the 12 patients, who then underwent further exploration of the resection cavity. At the time of the last postoperative follow-up, $11(92 \%)$ of the 12 patients were seizure free (Engel Class I). No patients underwent reoperation following iMRI-guided surgery.

CONCLUSIONS IMRI-guided resection of FCD in pediatric patients precluded the need for repeat surgery. Furthermore, it resulted in the achievement of complete resection in all the patients, leading to a high rate of postoperative seizure freedom.

http://thejns.org/doi/abs/10.3171/2015.10.PEDS15512

KEY WORDS intraoperative MRI; focal cortical dysplasia; epilepsy surgery; pediatric

$\mathrm{F}$ OCAL cortical dysplasia (FCD) is a common cause of intractable epilepsy in children, thus necessitating surgery. Factors that influence postoperative seizure outcome include seizure type, location, histopathology, multiplicity, age of the patient, and relationship to the eloquent cortex. However, meta-analysis has shown that the most significant predictor of postoperative seizure freedom is completeness of resection of the dysplastic cortical tissue. ${ }^{2,6,7,15,19}$ Gross complete extirpation of the lesion is, however, limited by difficulty in intraoperative identification of FCD tissue.

The application of intraoperative MRI (iMRI) as a safe and effective tool for real-time assistance in intractable epilepsy surgery has previously been demonstrated. $3,8,9,21,24,25$ Our objective in this study was to assess the impact of iMRI on intraoperative evaluation, surgical course of re-

ABBREVIATIONS ECOG = electrocorticography; $F C D$ = focal cortical dysplasia; $i M R I$ = intraoperative MRI; OE = operative event; $O R=$ operating room; SPGR = spoiled gradient echo recalled; $3 \mathrm{DGM}=3 \mathrm{D}$ gyral map.

SUBMITTED August 21, 2015. ACCEPTED October 29, 2015.

INCLUDE WHEN CITING Published online February 26, 2016; DOI: 10.3171/2015.10.PEDS15512. 
section, and seizure control outcome in children with intractable epilepsy due to FCD. This report is among the first to address the technique and outcomes following the utilization of iMRI for real-time intraoperative guidance in the resection of FCD.

\section{Methods}

The medical records and epilepsy protocol brain MR images of 12 consecutive pediatric patients undergoing surgery for intractable epilepsy due to FCD between January 2014 and April 2015 were retrospectively reviewed. Data collected included demographics, duration of the operative event (OE), use of iMRI, postoperative seizure freedom, and complications. Postsurgical seizure outcome was assessed utilizing the Engel Epilepsy Surgery Outcome Scale at the last postoperative follow-up visit. ${ }^{4}$ The preoperative, intraoperative, and postoperative images obtained in this study were reviewed by the attending neurosurgeon and neuroradiologist. We retrospectively reviewed these images and radiology reports for number of intraoperative scans, number of sequences acquired per scan, types of sequences acquired, and completeness of resection. This study was approved by the institutional review board of the Children's National Medical Center.

\section{Operative Setup and Surgical Technique}

Intraoperative neuronavigation (Brainlab and StealthStation navigation system, Medtronic Sofamor Danek) was used in lesion localization and craniotomy planning in all patients. After the craniotomy was performed, gyral recognition and localization of the sulcus within which the FCD was located (for bottom-of-the-sulcus FCD) was undertaken using both intraoperative neuronavigation and 3D image renditions of the cortical gyri (Fig. 1). These "3D gyral maps" (3DGMs) were obtained by postprocessing of the patient's preoperative brain images using skull stripping software. The 3DGM helped provide a global overview of the relationship of this gyrus or sulcus to adjacent gyri and sulci. Visual pattern recognition of this relationship provides a backup to operative neuronavigation since brain shift may have occurred after the craniotomy is performed.

Intraoperative electrocorticography (ECoG) using subdural strips, grids, or depth electrodes was then performed if it had preoperatively been determined as indicated. Generally, ECoG using a subdural grid or strips was performed for gyral lesions or bottom-of-sulcus FCDs located at reasonably superficial locations. For deeper lesions, depth electrodes were used, except in the insula (due to a higher risk of vascular injury) and in eloquent cortex. However, the decision regarding the site for resection was weighted more toward the structural location of the lesion rather than the ECoG findings.

Resection of the identified area of cortical dysplasia was then performed using standard microsurgical techniques. Once the surgeon felt that complete resection of the lesion had been achieved, the patient was transferred to the 1.5-T iMRI system (Greenline Achieva Nova Dual, Philips Medical System). Our iMRI system is located in an adjoining suite to a dedicated operating room (OR).
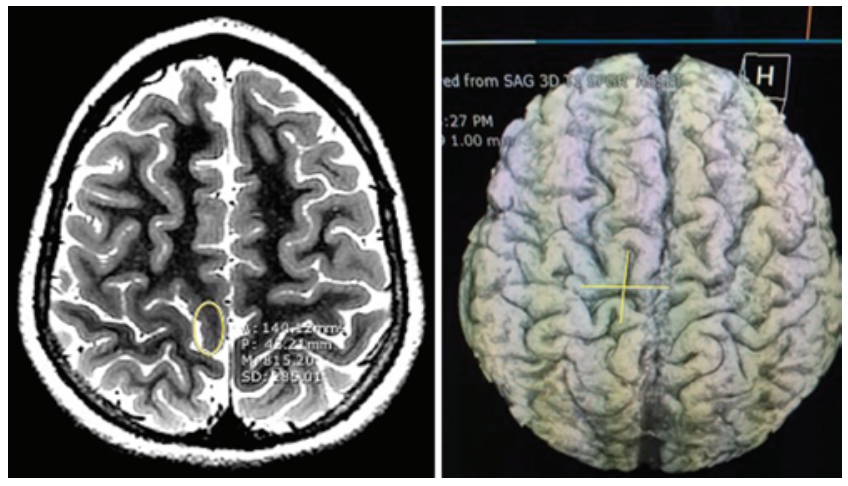

FIG. 1. Representative example of a 3DGM attained from preoperative MRI. Left: Spatially accurate multimodal images for intraoperative navigation are produced from the data. Right: 3DGM marking the precise localization of a sulcus. Figure is available in color online only.

The design of this OR incorporates standard MR safety features. The patient is positioned on a dedicated MRIcompatible operating table, which is rolled into the MR suite under maintenance of a sterile field. The duration of the preparation for transfer, image acquisition time, return to the OR, and resumption of surgery (if indicated) is approximately 45-60 minutes. The intraoperative sequences included a T1-weighted 3D spoiled gradient echo recalled (SPGR) (TE $3.2 \mathrm{msec}$, TR $6.7 \mathrm{msec}$, matrix $240 \times 240$, FOV $300 \mathrm{~mm}$, slice thickness $1.6 \mathrm{~mm}$, slab $34 \mathrm{~cm}$ ), T2weighted turbo spin echo (TE $100 \mathrm{msec}$, TR $4019 \mathrm{msec}$, matrix $296 \times 225$, FOV $280 \mathrm{~mm}$, slice thickness $3 \mathrm{~mm}$ ), and diffusion-weighted imaging sequence (TE $103 \mathrm{~mm}$, TR 5096 mm, matrix size $152 \times 106$, FOV 230 mm, slice thickness $4 \mathrm{~mm}$ ). T1-weighted axial or sagittal 3D SPGR volumetric images were each reformatted to coronal, sagittal, or axial format. The images were then reviewed independently by the attending pediatric neuroradiologist, looking for areas of residual dysplastic tissue. If evidence of residual FCD tissue was observed, the patient was returned to the OR for further resective surgery. In this situation, the Brainlab neuronavigation software was updated using the most recently acquired intraoperative 3D SPGR volumetric images. This ensured that intraoperative brain shift was accounted for. Surgery was then continued until a complete extirpation of the lesion was achieved as evidenced by another iMRI scan, unless precluded by intraoperative safety concerns.

Duration of the OE was recorded as the time of the patient's entry into the OR suite until exit from the OR suite. Hence, in addition to surgical time, this encompassed time for anesthesia, placement of peripheral lines, prepping of the patient, acquisition of all MR images, and anesthesia recovery. On average, these extra surgical activities took up to 2-3 hours. We felt it was relevant to include these extraoperative components because they are a reflection of the complexity of coordination of care and, therefore, represent indirect costs in undertaking this type of procedure.

\section{Results}

Twelve consecutive pediatric patients ( 8 females and 4 males) underwent iMRI-guided resection for FCD lesions; clinical characteristics are presented in Table 1. The age 
TABLE 1. Characteristics of patients undergoing iMRI-assisted FCD resection

\begin{tabular}{rrrrlllll}
\hline $\begin{array}{c}\text { Case } \\
\text { No. }\end{array}$ & $\begin{array}{c}\text { Age (yrs), } \\
\text { Sex }\end{array}$ & $\begin{array}{c}\text { Duration of } \\
\text { Epilepsy (mos) }\end{array}$ & $\begin{array}{c}\text { Histopathology } \\
\text { Type }\end{array}$ & Location of FCD & $\begin{array}{c}\text { Eloquent } \\
\text { Cortex }\end{array}$ & $\begin{array}{c}\text { Further } \\
\text { Resection }\end{array}$ & Op Time (mins) & $\begin{array}{c}\text { Engel } \\
\text { Class }\end{array}$ \\
\hline 1 & $17, \mathrm{~F}$ & 84 & I & Lt temporal & Yes & No & 261 & I \\
\hline 2 & $7, \mathrm{M}$ & 60 & IIA & Rt insular & No & Yes & 508 & I \\
\hline 3 & $6, \mathrm{~F}$ & 12 & IIB & Lt temporoparietal & Yes & Yes & 382 & I \\
\hline 4 & $1, \mathrm{M}$ & 5 & NA & Rt parietal & Yes & No & 382 & I \\
\hline 5 & $3, \mathrm{~F}$ & 3 & I & Rt parietal & Yes & Yes & 482 & I \\
\hline 6 & $9, \mathrm{~F}$ & 114 & IIA & Rt insular & No & Yes & 432 & I \\
\hline 7 & $13, \mathrm{~F}$ & 84 & IIA & Rt parietal & Yes & No & 371 & I \\
\hline 8 & $18, \mathrm{M}$ & 150 & NA & Rt frontal & No & No & 301 & I \\
\hline 9 & $10, \mathrm{~F}$ & 3 & IIA & Lt parietal & Yes & No & 293 & I \\
\hline 10 & $8, \mathrm{M}$ & 60 & NA & Rt parietal & No & Yes & 427 & I \\
\hline 11 & $2, \mathrm{~F}$ & 26 & IIB & Rt frontal & No & No & 280 & I \\
\hline 12 & $7, \mathrm{~F}$ & 74 & IIA & Rt insular & Yes & No & 361 & III \\
\hline
\end{tabular}

$\mathrm{NA}=\mathrm{FCD}$ of undetermined classification.

of onset ranged from birth to 9 years (mean 2.8 years), age at surgery ranged from 0.7 to 18.8 years (mean 8.8 years), and duration of seizures ranged from 3 to 150 months (mean 56.3 months). The mean ( \pm SEM) duration of follow-up was $3.5 \pm 1.0$ months (Table 2). Histopathological analysis of cortical specimens revealed that 2 patients had Type 1 FCD, 5 patients had Type $2 \mathrm{~A}$ FCD, 2 patients had Type $2 \mathrm{~B}$ FCD, and 3 patients had FCD of undetermined classification.

In most cases, the FCD had an extratemporal location: insular $(n=3)$, temporoparietal $(n=1)$, parietal $(n=5)$, and frontal $(n=2)$. The dysplastic tissue was located in eloquent cortex (sensory, motor, and language) in 7 of the 12 patients. Seven of the 12 cases involved reoperations of previous partial resections.

The duration of $\mathrm{OE}$ ranged from 261 minutes to 508 minutes (mean $373 \pm 23.0$ minutes). Total acquisition time of iMRI sequences (T1-weighted 3D SPGR, T2-weighted turbo spin echo, and diffusion-weighted imaging) ranged from 7 to 39 minutes (mean $18.9 \pm 3.0$ minutes) (this is different from the duration for preparation for transfer of the patient to the iMRI suite, image acquisition time, return to the OR, and resumption of surgery, which is approximately between 45 and 60 minutes). A single iMRI scan series was performed in 9 of 12 patients, with 3 cases $(2,5$, and 6) requiring a second series of acquisitions (Table 3 ).

A total of 41 iMRI images were acquired (mean per case $3.4 \pm 0.5$ images). In 5 (42\%) of 12 patients (Cases 2, $3,5,6$, and 10), iMRI images indicated residual dysplastic tissue, and these patients were returned to the OR for reexploration of the resection cavity. This led to further resection in 4 of the 5 patients (Cases 2, 5, 6, and 10). Confirmation of complete resection after lengthened surgery was performed by a second series of iMRI or postoperative MRI. Overall, we were able to achieve gross-total resection of dysplastic cortical tissue in all 12 patients in our cohort, which was confirmed by 1.5-T iMRI or 3-T postoperative MRI. No patients required a return to surgery at a later date for reoperation of residual dysplastic tissue.

At the time of the last postoperative follow-up, 11 (92\%) of the 12 patients in our cohort were seizure free
(Engel Class I). Worthwhile improvement (Engel Class III) was noted in 1 patient (Case 12). Moreover, in each of the 5 cases in which intraoperative imaging impacted the course of the surgery, the patient went on to be completely seizure free.

There were no reported accidents associated with iMRI utilization in our cohort. No patients experienced adverse effects associated with intraoperative ferromagnetic exposure, infection, or prolonged anesthesia. Postsurgical neurological complications included expected transient hemiparesis in 4 patients (Cases 4, 5, 7, and 9), all of whom were patients who had FCD lesions in the motor cortex. Expected transient alteration of sensation in the lower extremity occurred in 1 patient (Case 7) whose lesion was in the central sulcus and extended to the sensory cortex.

\section{Discussion}

In our cohort of 12 pediatric patients who had undergone iMRI-assisted resection of FCD, we observed a high rate of postoperative seizure freedom. We demonstrate that iMRI provided both near real-time assurance of completeness of resection and prevented later-date returns to the OR for resection of residual lesions. We present evidence to support the use of iMRI guidance in the OR to improve rates of complete resection and achieve superior seizure-freedom outcomes in patients with FCD.

We previously reported that the most significant predictor of postsurgical seizure freedom in children undergoing resection of FCD is completeness of resection. ${ }^{15}$ However, intraoperative detection of epileptogenic cortical dyplastic tissue remains a challenge, often precluding a com-

TABLE 2. Descriptive statistics of patient clinical parameters

\begin{tabular}{lc}
\hline \multicolumn{1}{c}{ Parameter } & Mean \pm SEM \\
\hline Age at op (yrs) & $8.8 \pm 1.6$ \\
\hline Duration of seizure (mos) & $56.3 \pm 3.8$ \\
\hline Age at seizure onset (mos) & $2.8 \pm 1.0$ \\
\hline Duration of follow-up (mos) & $3.5 \pm 1.0$ \\
\hline
\end{tabular}


TABLE 3. IMRI acquisition details for patients who underwent reexploration of the resection cavity following iMRI

\begin{tabular}{|c|c|c|c|c|c|}
\hline \multirow[b]{2}{*}{ Case No. } & \multicolumn{2}{|l|}{ 1st iMRI Sequence } & \multicolumn{2}{|l|}{ 2nd iMRI Sequence } & \multirow[b]{2}{*}{ Postop Imaging } \\
\hline & Description & Result & Description & Result & \\
\hline 2 & $\begin{array}{l}\text { T1 3D SPGR, T2 TSE axial, T2 TSE } \\
\text { coronal, DWI }\end{array}$ & I & $\begin{array}{l}\text { T1 3D SPGR, T2 TSE axial, T2 TSE } \\
\text { coronal, DWI }\end{array}$ & I & C \\
\hline 3 & $\begin{array}{l}\text { T1 3D SPGR, T2 TSE 3D, T2 TSE } \\
\text { coronal }\end{array}$ & $C^{*}$ & NP & - & - \\
\hline 5 & T1 3D SPGR, T2 TSE axial & I & T1 3D SPGR, T2 TSE axial & C & - \\
\hline 6 & T1 3D SPGR, T2 TSE axial, DWI & I & T1 3D SPGR, T2 TSE axial & C & - \\
\hline 10 & $\begin{array}{c}\text { T1 3D SPGR, T2 TSE axial, T2 TSE } \\
\text { coronal, T2 TSE sagittal, DWI }\end{array}$ & I & $\mathrm{NP}$ & - & C \\
\hline
\end{tabular}

$\mathrm{C}=$ complete resection; $\mathrm{DWI}=$ diffusion-weighted imaging; I = incomplete resection; NP = not performed; TSE = turbo spin echo; T1 = T1weighted; $\mathrm{T} 2$ = T2-weighted.

* Neuroradiologist reported "There is small amount of questionable residual abnormal cortex at the anterior and deep margin of the new resection." Upon further intraoperative exploration it was confirmed that abnormal tissue in the lesional area had been completely resected.

plete extirpation and resulting in unnecessary repeat operations. Neuronavigation with iMRI guidance has been previously reported to aid in increased rates of complete resection and seizure freedom in temporal and extratemporal lobe epilepsy. ${ }^{17,24}$ Drawing on the established role of iMRI guidance in tumor resections, our center began utilizing 1.5-T MRI intraoperatively for resection of FCD in January 2014.

iMRI images obtained in this cohort detected residual tissue, impacting surgical decision making in almost half of our cases. This resulted in further resection in 4 of those cases $(2,5,6$, and 10$)$, leading to complete resection, as confirmed by a second iMRI scan (Cases 5 and 6 ) or postoperative MRI (Cases 2 and 10). Thus, the utilization of iMRI increased our overall completeness of resection rate by one-third. Moreover, each of these patients achieved seizure freedom postoperatively, and none has required further reoperation.

We use Case 6 to illustrate the capacity of iMRI to guide the real-time decision making of the surgical approach for FCD resections. This patient presented with intractable epilepsy secondary to an FCD located in the right posterior insula and the posterior aspect of the right sylvian fissure (Fig. 2A). Resection was carried out until the surgeon felt a sufficient radical resection was performed, at which point iMRI images were obtained. It should be noted that during the surgery, this area of cortical dysplasia did not appear grossly distinct (from a visual or tactile perspective) from the rest of the cortex, so it was impossible to define margins. iMRI showed a small residual lesion just anterior to the resection cavity (Fig. 2B). The patient was returned to the OR, and further resection was carried out in the depths of the sulcus and anterior to the resection cavity until it was felt that complete resection had been achieved. Another iMRI scan was then performed, which confirmed a complete resection (Fig. 2C). The patient remains seizure free since surgery.

Improvement in postoperative seizure outcome for patients presenting with perirolandic FCD is linked to completeness of resection. ${ }^{20}$ The utility of iMRI guidance for resection of epileptogenic lesions in regions of eloquent cortex has been previously documented. ${ }^{24}$ This was also demonstrated in our patients. For example, the patient in
Case 5 presented with intractable epilepsy secondary to partial resection of parietal FCD localized in the perirolandic region (Fig. 3A). Resection of dysplastic tissue within the depths of the rolandic sulcus was carried out under the guidance of preoperative neuronavigation trajectory guidance. At this point, the surgeon used iMRI to define the extent of the resection. Residual tissue was identified immediately overlying the internal capsule (Fig. 3B). Further resection of the residual abnormal cortical tissue was undertaken with caution toward immediately adjacent eloquent cortex. At this point, the surgeon felt that a full resection had been accomplished, and a second iMRI confirmed complete resection (Fig. 3C). The patient is reported to be seizure free postoperatively. Transient right hemiparesis occurred immediately following surgery but was resolved within 2 days. The addition of iMRI to existing protocols for resection of FCD lesions in close proximity to eloquent cortex may thus aid in the completeness of resection, while preserving important functional capacities. As has been shown in resection of gliomas localized to eloquent cortex, we found the combination of iMRI with intraoperative neurophysiological monitoring to be critical to attaining a balance between achieving a complete resection, while not being unduly aggressive and causing postoperative neurological deficits to language, motor, or speech capacity. ${ }^{22}$

We found iMRI to be a useful part of the surgical armamentarium that enhanced intraoperative decision making. Despite this advantage, one of the patients in our cohort had a poor seizure-control outcome. The patient in Case 12 presented with intractable epilepsy secondary to FCD in the right posterior superior insula (Fig. 4A). Resection was carried out until the surgeon felt all lesional tissue had been removed, at which point iMRI was performed, confirming a complete resection (Fig. 4B). At the postoperative Day-42 and Day-74 follow-ups, the patient reported having seizures on multiple days resembling her preoperative seizures. 3-T MRI obtained on postoperative Day 74 further confirmed a complete resection (Fig. 4C). While the etiology of these recurring seizures is unknown, this case presents 2 important concerns of iMRI utilization. In our study, iMRI was obtained from a 1.5-T unit, which has an inferior resolution compared with a 3-T unit. ${ }^{14} \mathrm{Ad}-$ 

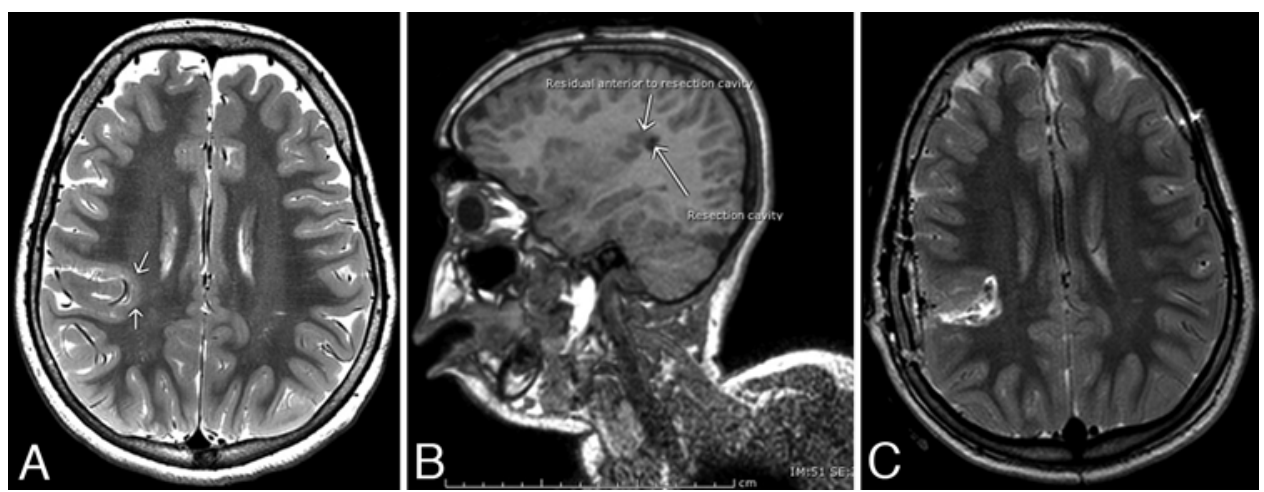

FIG. 2. Case 6. MR images obtained in a 9-year-old girl presenting with intractable epilepsy secondary to an FCD. A: Preoperative T2-weighted MR image showing an area of blurring of the gray-white matter margin, suggestive of an FCD deep within the right posterior sylvian fissure (arrows). B: Sagittal reconstruction of T1-weighted SPGR MR image obtained intraoperatively showing residual cortical dysplasia tissue just anterior to the resection cavity in the right posterior insula (arrows). C: Intraoperative T2-weighted MR image confirming complete resection of the area of FCD.

ditionally, surgical trauma, swelling, and blood products have the ability to distort intraoperative imaging, making it hard to discern areas of residual dysplastic tissue. Finally, this points to the limits of resolution of MRI, which is about $1 \mathrm{~mm} \cdot{ }^{13}$ Thus, better tools for the identification of the structural substrate of epileptogenesis (in this case, the area of cortical dysplasia) are indicated. In this regard, the use of optical coherence tomography (OCT) has been proposed, since OCT can improve this structural resolution to about 10 microns. $^{18}$

In our study, seizure freedom (Engel Class I) was achieved in over $90 \%$ of patients through the duration of follow-up. A literature review of seizure freedom following non-iMRI-guided resection of FCD yielded a range between $46 \%$ and $67 \% .{ }^{1,5,6,10-12,16,23}$ Our results demonstrate that by improving rates of gross-total resection of cortical dysplasia tissue, the addition of intraoperative imaging to existing epilepsy surgery techniques results in improved rates of seizure freedom and reduces the need for reoperation. Further randomized and blinded studies should com- pare the utilization of iMRI to standard epilepsy surgery protocols, addressing the variables of operative time, postsurgical outcomes, and morbidity. Moreover, one of the greatest assets of intraoperative imaging is the ability to confirm completeness of resection in near real time, thus reducing the potential need for extended hospital stays, postsurgical complications, and the need for reoperation. The health economics of the addition of iMRI use in FCD resections should, thus, be analyzed in comparison with established standard protocols.

This study is limited by a relatively small cohort of 12 patients, presented at a single center and reviewed retrospectively. To address this point, a randomized prospective multiple-center study of a larger cohort may further support the efficacy of iMRI guidance for FCD resection. Completeness of resection while using iMRI was assessed with a 1.5-T scanner. The limitations of spatial resolution in the 1.5-T scanner compared with a 3.0-T scanner may have impacted our assessment. Mean duration of postsurgical follow-up for our study was relatively short at 3.5
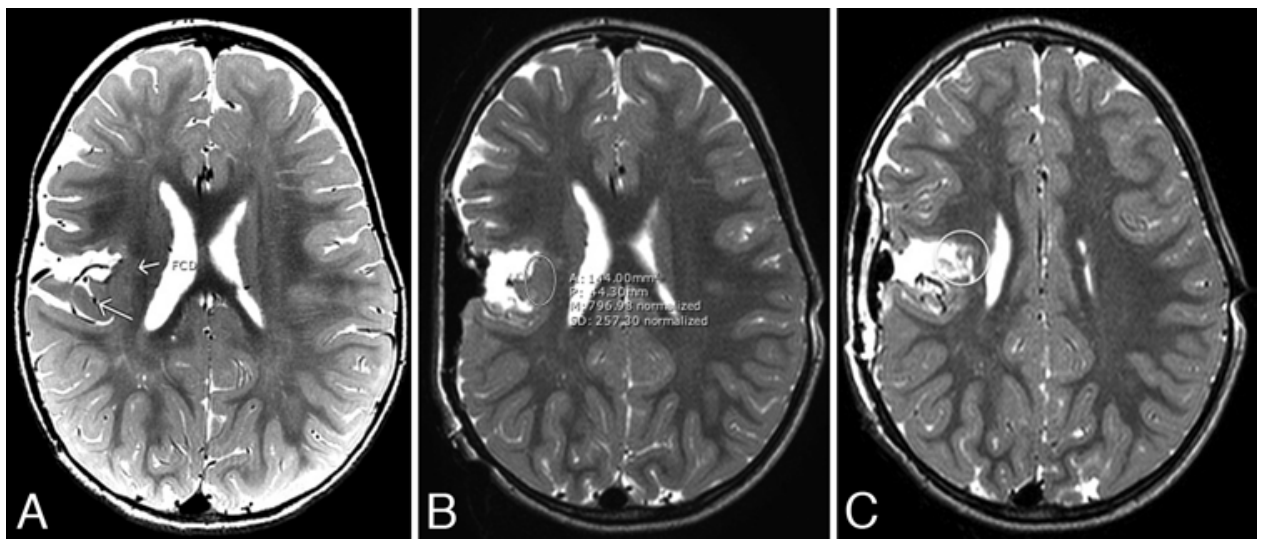

FIG. 3. Case 5. MR images obtained in a 3-year-old girl presenting with intractable epilepsy secondary to partial resection of an FCD. A: Preoperative T2-weighted MR image showing an area of FCD in the depths of the right central sulcus just adjacent to the internal capsule (arrows). B: Intraoperative T2-weighted axial MR image showing a residual area of FCD just adjacent to the internal capsule (circled area). C: Intraoperative T2-weighted axial MR image showing complete resection of the area of cortical dysplasia (circled area). 

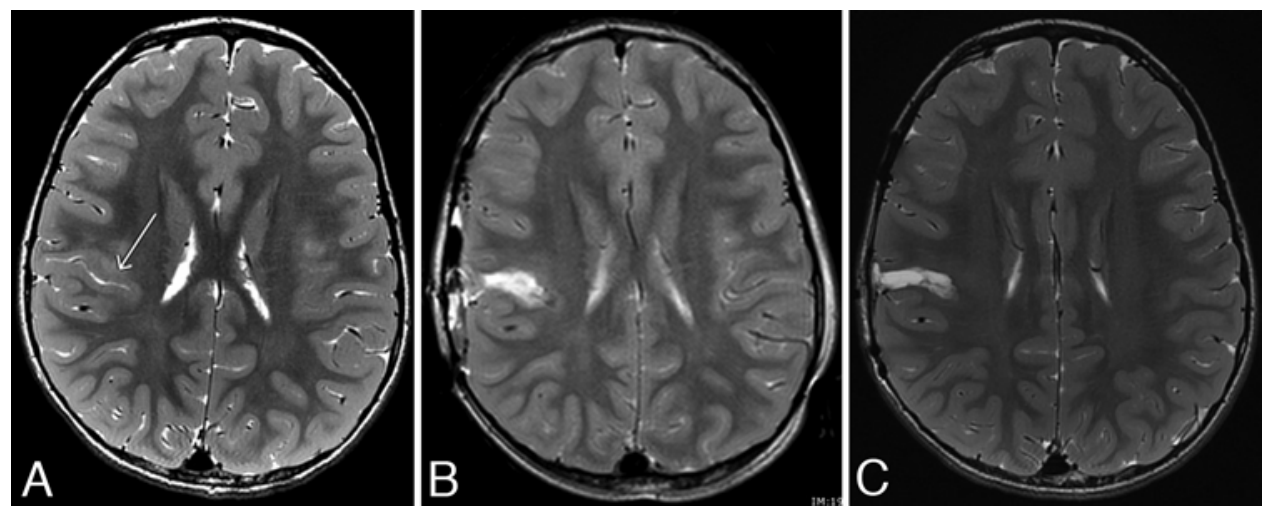

FIG. 4. Case 12. MR images obtained in a 7-year-old girl presenting with intractable epilepsy secondary to an FCD. A: Preoperative T2-weighted MR image showing an area of FCD in the posterior aspect of the right insula (arrow). B: Intraoperative T2weighted axial MR image showing complete resection of the area of cortical dysplasia. C: Postoperative T2-weighted MR image (3T) obtained on postoperative Day 74 confirming complete resection of the area of cortical dysplasia.

months. Further studies need to assess long-term outcomes of seizure freedom in patients undergoing iMRI-guided resections.

\section{Conclusions}

iMRI-guided resection of FCD in pediatric patients resulted in a high rate of postoperative seizure freedom and eliminated the need for repeat surgery. It enabled detection of residual FCD in near real time, thus ensuring complete resection in the same operative session. Our results suggest that the addition of iMRI to existing epilepsy surgery techniques and protocols can refine intraoperative decision making, leading to high rates of completeness of resection and improved seizure-control outcomes. Importantly, iMRI should be viewed as complementary to existing time-proven neurosurgical assistive technologies, including neuronavigation, ECoG, and neurophysiological monitoring.

\section{References}

1. Alexandre V Jr, Walz R, Bianchin MM, Velasco TR, TerraBustamante VC, Wichert-Ana L, et al: Seizure outcome after surgery for epilepsy due to focal cortical dysplastic lesions. Seizure 15:420-427, 2006

2. Cossu M, Lo Russo G, Francione S, Mai R, Nobili L, Sartori I, et al: Epilepsy surgery in children: results and predictors of outcome on seizures. Epilepsia 49:65-72, 2008

3. Cui ZQ, Ling ZP, Song HF, Hu S, Sun GC, Chen XL, et al: Combining pyramidal tract mapping, microscopic-based neuronavigation, and intraoperative magnetic resonance imaging improves outcome of epilepsy foci resection in the sensorimotor cortex. Turk Neurosurg 24:538-545, 2014

4. Engel J, Van Ness PC, Rasmussen TB, Ojemann LM: Outcome with respect to epileptic seizures, in Engel J, (ed): Surgical Treatment of the Epilepsies, ed 2. New York: Raven Press, 1993, pp 609-621

5. Fauser S, Essang C, Altenmüller DM, Staack AM, Steinhoff BJ, Strobl K, et al: Long-term seizure outcome in 211 patients with focal cortical dysplasia. Epilepsia 56:66-76, 2015

6. Fountas KN, King DW, Meador KJ, Lee GP, Smith JR: Epilepsy in cortical dysplasia: factors affecting surgical outcome. Stereotact Funct Neurosurg 82:26-30, 2004

7. Hirabayashi S, Binnie CD, Janota I, Polkey CE: Surgical treatment of epilepsy due to cortical dysplasia: clinical and EEG findings. J Neurol Neurosurg Psychiatry 56:765-770, 1993

8. Jankovski A, Francotte F, Vaz G, Fomekong E, Duprez T, Van Boven M, et al: Intraoperative magnetic resonance imaging at 3-T using a dual independent operating room-magnetic resonance imaging suite: development, feasibility, safety, and preliminary experience. Neurosurgery 63:412-426, 2008

9. Kaibara T, Myles ST, Lee MA, Sutherland GR: Optimizing epilepsy surgery with intraoperative MR imaging. Epilepsia 43:425-429, 2002

10. Kim DW, Lee SK, Chu K, Park KI, Lee SY, Lee CH, et al: Predictors of surgical outcome and pathologic considerations in focal cortical dysplasia. Neurology 72:211-216, 2009

11. Kloss S, Pieper T, Pannek H, Holthausen H, Tuxhorn I: Epilepsy surgery in children with focal cortical dysplasia (FCD): results of long-term seizure outcome. Neuropediatrics 33:21-26, 2002

12. Krsek P, Maton B, Jayakar P, Dean P, Korman B, Rey G, et al: Incomplete resection of focal cortical dysplasia is the main predictor of poor postsurgical outcome. Neurology 72:217-223, 2009

13. Lüsebrink F, Wollrab A, Speck O: Cortical thickness determination of the human brain using high resolution $3 \mathrm{~T}$ and $7 \mathrm{~T}$ MRI data. Neuroimage 70:122-131, 2013

14. Mellerio C, Labeyrie MA, Chassoux F, Roca P, Alami O, Plat $\mathrm{M}$, et al: 3T MRI improves the detection of transmantle sign in type 2 focal cortical dysplasia. Epilepsia 55:117-122, 2014

15. Oluigbo CO, Wang J, Whitehead MT, Magge S, Myseros JS, Yaun A, et al: The influence of lesion volume, perilesion resection volume, and completeness of resection on seizure outcome after resective epilepsy surgery for cortical dysplasia in children. J Neurosurg Pediatr 15:644-650, 2015

16. Park CK, Kim SK, Wang KC, Hwang YS, Kim KJ, Chae JH, et al: Surgical outcome and prognostic factors of pediatric epilepsy caused by cortical dysplasia. Childs Nerv Syst 22:586-592, 2006

17. Roessler K, Sommer B, Grummich P, Coras R, Kasper BS, Hamer HM, et al: Improved resection in lesional temporal lobe epilepsy surgery using neuronavigation and intraoperative MR imaging: favourable long term surgical and seizure outcome in 88 consecutive cases. Seizure 23:201-207, 2014

18. Roper SN, Moores MD, Gelikonov GV, Feldchtein FI, Beach NM, King MA, et al: In vivo detection of experimentally induced cortical dysgenesis in the adult rat neocortex using optical coherence tomography. J Neurosci Methods 80:9198, 1998 
19. Rowland NC, Englot DJ, Cage TA, Sughrue ME, Barbaro NM, Chang EF: A meta-analysis of predictors of seizure freedom in the surgical management of focal cortical dysplasia. J Neurosurg 116:1035-1041, 2012

20. Sarkis RA, Jehi LE, Bingaman WE, Najm IM: Surgical outcome following resection of rolandic focal cortical dysplasia. Epilepsy Res 90:240-247, 2010

21. Schwartz TH, Marks D, Pak J, Hill J, Mandelbaum DE, Holodny AI, et al: Standardization of amygdalohippocampectomy with intraoperative magnetic resonance imaging: preliminary experience. Epilepsia 43:430-436, 2002

22. Senft C, Forster MT, Bink A, Mittelbronn M, Franz K, Seifert $\mathrm{V}$, et al: Optimizing the extent of resection in eloquently located gliomas by combining intraoperative MRI guidance with intraoperative neurophysiological monitoring. J Neurooncol 109:81-90, 2012

23. Siegel AM, Cascino GD, Meyer FB, Marsh WR, Scheithauer BW, Sharbrough FW: Surgical outcome and predictive factors in adult patients with intractable epilepsy and focal cortical dysplasia. Acta Neurol Scand 113:65-71, 2006

24. Sommer B, Grummich P, Coras R, Kasper BS, Blumcke I, Hamer HM, et al: Integration of functional neuronavigation and intraoperative MRI in surgery for drug-resistant extratemporal epilepsy close to eloquent brain areas. Neurosurg Focus 34(4):E4, 2013
25. Walker DG, Talos F, Bromfield EB, Black PM: Intraoperative magnetic resonance for the surgical treatment of lesions producing seizures. J Clin Neurosci 9:515-520, 2002

\section{Disclosures}

The authors report no conflict of interest concerning the materials or methods used in this study or the findings specified in this paper.

\section{Author Contributions}

Conception and design: Oluigbo, Sacino, Gaillard. Acquisition of data: Oluigbo, Sacino. Analysis and interpretation of data: Oluigbo, Sacino. Drafting the article: Oluigbo, Sacino. Critically revising the article: all authors. Reviewed submitted version of manuscript: Oluigbo, Sacino. Approved the final version of the manuscript on behalf of all authors: Oluigbo. Study supervision: Oluigbo, Gaillard.

\section{Correspondence}

Chima O. Oluigbo, Department of Neurosurgery, Children's National Medical Center, 111 Michigan Ave. NW, Washington, DC 20010. email: coluigbo@cnmc.org. 